\title{
Physical Work and Exercise Reduce the Risk of Cognitive Impairment in Rural Older Adults: A Population-based Longitudinal Study
}

Fan He

School of public health, Fudan University; Zhejiang Provincial Center for Disease Control and Prevention https://orcid.org/0000-0001-6027-7157

Junfen Lin

Zhejiang Provincial Center for Disease Control and Prevention

Fudong Li

Zhejiang Provincial Center for Disease Control and Prevention

Yujia Zhai

Zhejiang Provincial Center for Disease Control and Prevention

Tao Zhang

Zhejiang Provincial Center for Disease Control and Prevention

Xue Gu

Zhejiang Provincial Center for Disease Control and Prevention

Genming Zhao ( $\nabla$ gmzhao@shmu.edu.cn )

https://orcid.org/0000-0002-9625-478X

\section{Research article}

Keywords: physical activity; cognitive impairment; longitudinal study; hazard ratio; older adults

Posted Date: February 21st, 2020

DOI: https://doi.org/10.21203/rs.2.24173/v1

License: (c) (i) This work is licensed under a Creative Commons Attribution 4.0 International License. Read Full License

Version of Record: A version of this preprint was published at Current Alzheimer Research on November 18th, 2021. See the published version at https://doi.org/10.2174/1567205018666211118100451. 


\section{Abstract}

Background: The effect of physical work on the risk of cognitive impairment in Chinese older adults living in rural areas remains to be elucidated. We investigated whether physical work and exercise can reduce the risk of cognitive impairment. Methods: We collected data from 7,000 individuals without cognitive impairment (age $\geq 60$ years) over a follow-up period of 2 years. The Chinese version of the Mini-Mental State Examination was used to assess cognitive function, and the multivariable Cox regression model was used to identify associations between physical work/exercise and cognitive impairment. Results: Over a median follow-up period of 1.93 years, 1,224 (17.5\%) of 7,000 participants developed cognitive impairment, with a total incidence of 97.69 per 1,000 person-years. Participation in physical work (hazard ratio [HR]: 0.66; 95\% confidence interval [Cl], 0.55-0.78) or exercise (HR: 0.76; 95\% Cl, 0.62-0.93) was associated with a reduced risk of cognitive impairment. Agricultural work (HR: 0.60; 95\% Cl, 0.49-0.73) and walking/tai chi (HR: 0.75; 95\% Cl, 0.60-0.93) exerted significant protective effects against cognitive impairment. Conclusions: Physical work and exercise can reduce the risk of cognitive impairment in older adults. Reasonable types and appropriate intensities of physical activity are recommended to prevent or delay the progression of cognitive impairment.

\section{Background}

In 2017, there were 962 million individuals over the age of 60 worldwide, and this number is expected to more than double by 2050 and triple by 2100 [1]. Rapid growth in the population of older adults has presented severe challenges to the health care system, patients themselves, and their caregivers. In addition, the incidence of age-related diseases including neurodegenerative dementias such as Alzheimer's disease (AD) is expected to increase dramatically in the coming years, exerting a substantial impact on individual patients and society [2-5].

Accumulating evidence from epidemiological studies has illustrated the protective effect of physical activity against cognitive impairment and dementia in older adults [6-10]. In addition to enhancing hippocampal neurogenesis, synaptic plasticity, neurotrophin levels, and cardiovascular fitness, physical activity has been associated with greater white matter integrity and increased functional connectivity in the brain $[11,12]$. However, the definitions of physical activity have been inconsistent across studies, some of which focused on physical exercise alone. According to the World Health Organization, physical activity refers to any bodily movement produced by skeletal muscles that requires energy expenditure. Thus, physical activity encompasses exercise, any other physical activity conducted during leisure time, and work-related physical activities [13].

Previous studies have projected that the number of people over the age of 60 in China will increase to approximately 255 million, accounting for $17.8 \%$ of the total population, by 2020 [14]. At present, nearly half of older adults live in rural areas. Thus, participating in physical work, especially agricultural work, represents the main form of physical activity in this population. However, few studies have investigated the independent and interactive effects of physical work and exercise on the risk of cognitive impairment in Chinese older adults living in rural areas.

Therefore, in the current large-scale, population-based study, we aimed to explore the relationships among physical work, physical exercise, and the risk of cognitive impairment in Chinese older adults over a 2-year follow-up period.

\section{Methods}

\subsection{Participants}

All participants were enrolled in the Zhejiang Major Public Health Surveillance (ZJMPHS) Program, a prospective study of health issues and related factors among older adults. The program was conducted in rural and suburban areas of 
Zhejiang province, which is an economically developed province located in the Yangtze river delta on the southeastern coast of China. It has a humid monsoon climate with abundant light and rainfall and a resident population of 57.37 million (10.8 million people over the age of 60 ). Detailed information about the program has been described in previous reports [15]. At baseline (2014), six counties were randomly selected from the 90 counties in Zhejiang province. Then, one town in each county and several villages/communities in each town were randomly selected. All permanent residents aged $\geq 60$ years in these selected communities were recruited. Finally, we interviewed a total of 9,353 of the 9,425 eligible residents. Data regarding questionnaires, scales, and physical examinations (including height, weight, waist circumference, hip circumference, blood pressure and heart rate) were obtained by trained community general practitioners in community hospitals or by visiting the participants at their homes. Because we were interested in whether physical work and exercise are associated with the prospective risk of developing cognitive impairment, we excluded participants with cognitive impairment at baseline. Among the 9,353 participants, 7,947 exhibited no cognitive impairment at baseline. During the 2-year follow-up period, 447 (5.6\%) participants died, while 500 (6.3\%) were unavailable for follow-up. The remaining 7,000 participants who completed cognitive function tests at baseline and follow-up constituted the analytic cohort of this study. For missing values, the practitioner was instructed to conduct a timely re-interview or supplement the data at the next follow-up. Participation was voluntary, and written informed consent was obtained from each person. The program was approved by the Ethics Committee of the Zhejiang Provincial Center for Disease Control and Prevention.

\subsection{Assessment of Physical Work and Exercise}

Physical activity in this study included two aspects: physical work and physical exercise. For investigation of physical work, participants first reported whether they had engaged in agricultural work (including farming, forestry, animal husbandry, and fishery) in recent years. Those who did not participate in agricultural work further reported whether they had participated in other forms of physical activity and the type of activity performed. We collected data regarding retirement status, housework, unemployment, physical disabilities, sedentary activities (e.g., knitting, sewing, etc.), work performed while standing (e.g., janitorial work, retail work, etc.), light physical work (e.g., carpentry, electrical work, etc.), and intense physical work (e.g., mining, loading/construction, etc.). Participants also reported the type of transportation taken to work (walking, bicycle, motorcycle, car, or bus).

Because physical exercise may exert a significant protective effect on cognitive function, we considered the interactions between agricultural work and physical exercise. Thus, we asked participants to report their physical exercise habits over the past year, including type (walking/tai chi, brisk walking/yangko, running/aerobics, public square dancing, etc.) and intensity (frequency and duration).

\subsection{Assessment of Cognitive Function}

We used the Chinese version of the Mini-Mental State Examination (MMSE) to assess cognitive function in each participant. The MMSE includes 30 items, and the maximum score is 30 . Higher MMSE scores indicate better cognitive function. We used the following, widely accepted education-specific cut-off points to define cognitive impairment: $17 / 18$ for those with no education, $20 / 21$ for those with primary education only, and $24 / 25$ for those with education beyond the primary level [16].

\subsection{Other Variables}

At baseline (2014), we collected demographic data regarding sex, ethnic group (Han, She, Miao, Hui, etc.), age, body mass index (BMI), education level (illiterate or semiliterate, primary school, junior high school, high school graduation or higher), marital status (married, unmarried, widowed, or divorced), job (never worked, farmer, housework, standard work, and others), and family income. 
The study also collected information regarding the following: living alone, eating alone, participation in group activities (never, occasionally, frequently), sleep quality (always poor, frequently poor, occasionally poor, always good), napping (never or occasionally, sometimes, frequently), smoking status (non-smokers, current smokers, and ex-smokers), passive smoking in the family, passive smoking in public places, alcohol consumption (non-drinkers, current drinkers, and ex-drinkers), tea consumption (non-drinkers, current drinkers, ex-drinkers), and intake of drinking water per day. We used patient medical records to assess the presence of underlying diseases (stroke, high blood pressure, hyperlipidemia, diabetes, coronary heart disease, chronic bronchitis, gallstones, tumor, arthritis, cataracts, and others).

\subsection{Statistical Analysis}

We compared the distributions of demographic variables, physical work, exercise, and other covariates between participants with and without cognitive impairment using univariate Cox regression analysis. We used multivariable Cox regression analyses to calculate adjusted hazard ratios (HRs) and 95\% confidence intervals (Cls) as measures of the associations among physical work, exercise, and cognitive impairment after controlling for sex, ethnicity, age, BMI, education, marital status, job type, family income, living or eating alone, participating in group activities, sleep quality, napping, smoking and passive smoking, alcohol consumption, tea consumption, water consumption and disease history. We utilized stratified analyses and interaction terms to examine the modulatory effects of exercise on the interaction between physical work and cognitive impairment after considering the above variables as confounding factors [17]. All analyses were performed using SPSS software version 20.0 (SPSS Inc., Chicago, IL, USA), statistical significance was set as at $p<0.05$, and the "enter" method was used when building multivariable models.

\section{Results}

The incidence of cognitive impairment was 97.69 per 1,000 person-years among older adults in Zhejiang, China. In this study, the incidence of cognitive impairment was higher among women (114.96 per 1,000 person-years) than men (79.73 per 1,000 person-years). The incidence of cognitive impairment in each age group $(<65,65-69,70-74,75-79$, 80-84, and $85+$ ) was as follows: $63.20,71.26,94.06,132.08,186.12$, and 213.90 per 1,000 person-years, respectively.

Among the 7,000 participants with normal cognitive function at baseline, 1,224 (17.5\%) developed cognitive impairment. Table 1 shows the characteristics of participants with normal cognitive function and those with cognitive impairment. The proportion of participants with cognitive impairment was higher among women, widows, those who had never worked or performed housework only, and those considered elderly. Those with cognitive impairment were more likely to have lower BMI (<18.50); education; or family income; and to live alone, eat alone, or never participate in group activities. Moreover, those with cognitive impairment appeared to exhibit poorer sleep quality, a lower frequency of napping, and tended to be non-smokers/non-drinkers of tea or alcohol. Our results also suggested that cognitive impairment was associated with certain underlying diseases, such as stroke and hyperlipidemia. 
Table 1

Characteristics of participants with normal cognitive function and cognitive impairment based on selected covariates.

\begin{tabular}{|c|c|c|c|c|c|c|c|}
\hline & \multicolumn{2}{|c|}{ Cognitive impairment } & \multicolumn{2}{|c|}{$\begin{array}{l}\text { Normal cognitive } \\
\text { function }\end{array}$} & \multirow[t]{2}{*}{ HR } & \multirow[t]{2}{*}{$95 \% \mathrm{Cl}$} & \multirow[t]{2}{*}{$P$ value } \\
\hline & No. & $\%$ & No. & $\%$ & & & \\
\hline \multicolumn{8}{|l|}{ Gender } \\
\hline Female & 757 & 21.1 & 2834 & 78.9 & 1.00 & Ref. & \\
\hline Male & 505 & 14.8 & 2904 & 85.2 & 0.67 & $0.60-0.75$ & $<0.001$ \\
\hline \multicolumn{8}{|l|}{ Ethnicity } \\
\hline She, Miao, Hui, etc. & 38 & 15.1 & 213 & 84.9 & 1.00 & Ref. & \\
\hline Han & 1224 & 18.1 & 5524 & 81.9 & 0.92 & $0.64-1.34$ & 0.676 \\
\hline \multicolumn{8}{|l|}{ Age (years) } \\
\hline$<65$ & 194 & 10.9 & 1580 & 89.1 & 1.00 & Ref. & \\
\hline $65-69$ & 284 & 13.2 & 1860 & 86.8 & 1.03 & $0.86-1.24$ & 0.757 \\
\hline $70-74$ & 231 & 17.9 & 1060 & 82.1 & 1.41 & $1.17-1.72$ & $<0.001$ \\
\hline $75-79$ & 229 & 25.1 & 685 & 74.9 & 1.95 & $1.61-2.37$ & $<0.001$ \\
\hline $80-84$ & 228 & 35.4 & 416 & 64.6 & 2.61 & $2.15-3.17$ & $<0.001$ \\
\hline$\geq 85$ & 96 & 41.2 & 137 & 58.8 & 3.28 & $2.56-4.20$ & $<0.001$ \\
\hline \multicolumn{8}{|l|}{$\mathrm{BMI}\left(\mathrm{kg} / \mathrm{m}^{2}\right)$} \\
\hline $18.5-24.99$ & 765 & 17.6 & 3588 & 82.4 & 1.00 & Ref. & \\
\hline$<18.50$ & 100 & 29.2 & 242 & 70.8 & 1.50 & $1.22-1.85$ & $<0.001$ \\
\hline$>24.99$ & 312 & 15.9 & 1649 & 84.1 & 0.99 & $0.87-1.13$ & 0.873 \\
\hline \multicolumn{8}{|l|}{ Education } \\
\hline Illiterate or semiliterate & 738 & 21.7 & 2657 & 78.3 & 1.00 & Ref. & \\
\hline Primary school & 454 & 14.7 & 2635 & 85.3 & 0.70 & $0.62-0.79$ & $<0.001$ \\
\hline Junior high school & 61 & 13.9 & 377 & 86.1 & 0.67 & $0.51-0.87$ & 0.003 \\
\hline High school graduation or higher & 9 & 11.5 & 69 & 88.5 & 0.58 & $0.30-1.12$ & 0.105 \\
\hline \multicolumn{8}{|l|}{ Marital status } \\
\hline Unmarried & 13 & 13.5 & 83 & 86.5 & 1.00 & Ref. & \\
\hline Married & 887 & 16.1 & 4628 & 83.9 & 1.55 & $0.90-2.70$ & 0.116 \\
\hline Widowed & 352 & 26.1 & 995 & 73.9 & 2.27 & $1.30-3.96$ & 0.004 \\
\hline Divorced & 6 & 20.7 & 23 & 79.3 & 1.71 & $0.65-4.52$ & 0.278 \\
\hline
\end{tabular}

HR: hazard ratio; Cl: confidence interval; BMI: body mass index. 


\begin{tabular}{|c|c|c|c|c|c|c|c|}
\hline & \multicolumn{2}{|c|}{ Cognitive impairment } & \multicolumn{2}{|c|}{$\begin{array}{l}\text { Normal cognitive } \\
\text { function }\end{array}$} & \multirow[t]{2}{*}{ HR } & \multirow[t]{2}{*}{$95 \% \mathrm{Cl}$} & \multirow[t]{2}{*}{$P$ value } \\
\hline & No. & $\%$ & No. & $\%$ & & & \\
\hline \multicolumn{8}{|l|}{ Job Type } \\
\hline Never worked & 425 & 25.2 & 1260 & 74.8 & 1.00 & Ref. & \\
\hline Farmers & 514 & 16.7 & 2562 & 83.3 & 0.45 & $0.39-0.53$ & $<0.001$ \\
\hline Housework & 164 & 20.0 & 658 & 80.0 & 0.46 & $0.37-0.56$ & $<0.001$ \\
\hline Workers & 87 & 11.8 & 649 & 88.2 & 0.39 & $0.30-0.49$ & $<0.001$ \\
\hline Others & 69 & 10.6 & 584 & 89.4 & 0.32 & $0.24-0.41$ & $<0.001$ \\
\hline \multicolumn{8}{|c|}{ 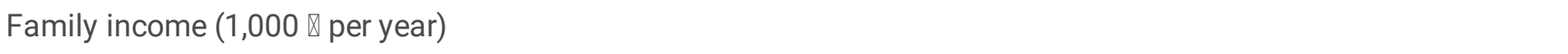 } \\
\hline$<10$ & 320 & 30.3 & 737 & 69.7 & 1.00 & Ref. & \\
\hline $10-19$ & 349 & 22.8 & 1185 & 77.2 & 0.73 & $0.62-0.85$ & $<0.001$ \\
\hline $20-49$ & 239 & 12.4 & 1690 & 87.6 & 0.41 & $0.34-0.49$ & $<0.001$ \\
\hline $50-99$ & 192 & 15.7 & 1031 & 84.3 & 0.45 & $0.37-0.55$ & $<0.001$ \\
\hline$\geq 100$ & 160 & 12.8 & 1090 & 87.2 & 0.27 & $0.22-0.34$ & $<0.001$ \\
\hline \multicolumn{8}{|l|}{ Living alone } \\
\hline No & 1023 & 17.1 & 4943 & 82.9 & 1.00 & Ref. & \\
\hline Yes & 239 & 23.1 & 795 & 76.9 & 1.30 & $1.13-1.51$ & $<0.001$ \\
\hline \multicolumn{8}{|l|}{ Eating alone } \\
\hline No & 1012 & 17.0 & 4927 & 83.0 & 1.00 & Ref. & \\
\hline Yes & 250 & 23.6 & 811 & 76.4 & 1.38 & $1.20-1.58$ & $<0.001$ \\
\hline \multicolumn{8}{|c|}{ Participating in group activities } \\
\hline Never & 911 & 21.3 & 3372 & 78.7 & 1.00 & Ref. & \\
\hline Occasionally & 262 & 14.3 & 1568 & 85.7 & 0.74 & $0.64-0.86$ & $<0.001$ \\
\hline Frequently & 89 & 10.1 & 794 & 89.9 & 0.51 & $0.41-0.64$ & $<0.001$ \\
\hline \multicolumn{8}{|l|}{ Sleep quality } \\
\hline Always poor & 64 & 19.3 & 267 & 80.7 & 1.00 & Ref. & \\
\hline Frequently poor & 172 & 19.9 & 692 & 80.1 & 0.98 & $0.74-1.31$ & 0.913 \\
\hline Occasionally poor & 334 & 16.1 & 1746 & 83.9 & 0.64 & $0.49-0.85$ & 0.002 \\
\hline Always good & 692 & 18.6 & 3028 & 81.4 & 0.86 & $0.66-1.11$ & 0.250 \\
\hline \multicolumn{8}{|l|}{ Napping } \\
\hline Never or occasionally & 726 & 18.8 & 3132 & 81.2 & 1.00 & Ref. & \\
\hline
\end{tabular}




\begin{tabular}{|c|c|c|c|c|c|c|c|}
\hline & \multicolumn{2}{|c|}{ Cognitive impairment } & \multicolumn{2}{|c|}{$\begin{array}{l}\text { Normal cognitive } \\
\text { function }\end{array}$} & \multirow[t]{2}{*}{ HR } & \multirow[t]{2}{*}{$95 \% \mathrm{Cl}$} & \multirow[t]{2}{*}{$P$ value } \\
\hline & No. & $\%$ & No. & $\%$ & & & \\
\hline Sometimes & 233 & 18.4 & 1032 & 81.6 & 1.16 & $0.99-1.35$ & 0.068 \\
\hline Frequently & 303 & 16.2 & 1569 & 83.8 & 1.10 & $0.96-1.27$ & 0.170 \\
\hline \multicolumn{8}{|l|}{ Smoking } \\
\hline Non-smokers & 979 & 19.7 & 3982 & 80.3 & 1.00 & Ref. & \\
\hline Current smokers & 194 & 14.3 & 1166 & 85.7 & 0.67 & $0.58-0.79$ & $<0.001$ \\
\hline Ex-smokers & 89 & 13.1 & 589 & 86.9 & 0.68 & $0.54-0.84$ & 0.001 \\
\hline \multicolumn{8}{|c|}{ Passive smoking in the family } \\
\hline No & 1111 & 18.1 & 5030 & 81.9 & 1.00 & Ref. & \\
\hline Yes & 151 & 17.6 & 708 & 82.4 & 1.24 & $1.04-1.48$ & 0.015 \\
\hline \multicolumn{8}{|c|}{ Passive smoking in public places } \\
\hline No & 1176 & 19.0 & 5025 & 81.0 & 1.00 & Ref. & \\
\hline Yes & 86 & 10.8 & 713 & 89.2 & 0.67 & $0.54-0.85$ & 0.001 \\
\hline \multicolumn{8}{|c|}{ Alcohol consumption } \\
\hline Non-drinkers & 918 & 19.5 & 3781 & 80.5 & 1.00 & Ref. & \\
\hline Current drinkers & 269 & 14.7 & 1559 & 85.3 & 0.75 & $0.65-0.86$ & $<0.001$ \\
\hline Ex-drinkers & 71 & 15.3 & 392 & 84.7 & 0.83 & $0.65-1.06$ & 0.139 \\
\hline \multicolumn{8}{|l|}{ Tea consumption } \\
\hline Non-drinkers & 1003 & 19.8 & 4059 & 80.2 & 1.00 & Ref. & \\
\hline Current drinkers & 239 & 13.4 & 1544 & 86.6 & 0.66 & $0.57-0.77$ & $<0.001$ \\
\hline Ex-drinkers & 13 & 9.9 & 118 & 90.1 & 0.54 & $0.31-0.94$ & 0.028 \\
\hline \multicolumn{8}{|c|}{ Water consumption (ml per day) } \\
\hline$\leq 500$ & 818 & 18.7 & 3564 & 81.3 & 1.00 & Ref. & \\
\hline $501-1000$ & 337 & 17.5 & 1590 & 82.5 & 0.90 & $0.79-1.03$ & 0.119 \\
\hline$>1000$ & 103 & 15.8 & 547 & 84.2 & 0.98 & $0.80-1.22$ & 0.879 \\
\hline \multicolumn{8}{|l|}{ Disease history } \\
\hline \multicolumn{8}{|l|}{ Stroke } \\
\hline No & 1212 & 17.8 & 5606 & 82.2 & 1.00 & Ref. & \\
\hline Yes & 50 & 27.8 & 130 & 72.2 & 1.46 & $1.10-1.94$ & 0.009 \\
\hline \multicolumn{8}{|c|}{ High blood pressure } \\
\hline
\end{tabular}




\begin{tabular}{|c|c|c|c|c|c|c|c|}
\hline & \multicolumn{2}{|c|}{ Cognitive impairment } & \multicolumn{2}{|c|}{$\begin{array}{l}\text { Normal cognitive } \\
\text { function }\end{array}$} & \multirow[t]{2}{*}{ HR } & \multirow[t]{2}{*}{$95 \% \mathrm{Cl}$} & \multirow[t]{2}{*}{$P$ value } \\
\hline & No. & $\%$ & No. & $\%$ & & & \\
\hline No & 678 & 18.3 & 3033 & 81.7 & 1.00 & Ref. & \\
\hline Yes & 583 & 17.7 & 2705 & 82.3 & 1.02 & $0.91-1.15$ & 0.687 \\
\hline \multicolumn{8}{|c|}{ Hyperlipidemia } \\
\hline No & 1230 & 18.4 & 5463 & 81.6 & 1.00 & Ref. & \\
\hline Yes & 32 & 10.7 & 268 & 89.3 & 0.86 & $0.60-1.23$ & 0.404 \\
\hline \multicolumn{8}{|c|}{ Diabetes } \\
\hline No & 1155 & 18.3 & 5153 & 81.7 & 1.00 & Ref. & \\
\hline Yes & 107 & 15.6 & 579 & 84.4 & 1.03 & $0.85-1.26$ & 0.743 \\
\hline \multicolumn{8}{|c|}{ Coronary heart disease } \\
\hline No & 1205 & 17.9 & 5534 & 82.1 & 1.00 & Ref. & \\
\hline Yes & 57 & 22.1 & 201 & 77.9 & 1.22 & $0.93-1.6$ & 0.144 \\
\hline \multicolumn{8}{|c|}{ Chronic bronchitis } \\
\hline No & 1229 & 17.9 & 5619 & 82.1 & 1.00 & Ref. & \\
\hline Yes & 33 & 22.4 & 114 & 77.6 & 1.50 & $1.06-2.13$ & 0.021 \\
\hline \multicolumn{8}{|c|}{ Gallstones } \\
\hline No & 1229 & 18.2 & 5522 & 81.8 & 1.00 & Ref. & \\
\hline Yes & 33 & 13.4 & 213 & 86.6 & 0.92 & $0.65-1.31$ & 0.654 \\
\hline \multicolumn{8}{|c|}{ Tumor } \\
\hline No & 1241 & 18.2 & 5595 & 81.8 & 1.00 & Ref. & \\
\hline Yes & 21 & 13.0 & 140 & 87.0 & 0.82 & $0.53-1.27$ & 0.376 \\
\hline \multicolumn{8}{|c|}{ Arthritis } \\
\hline No & 1218 & 18.0 & 5532 & 82.0 & 1.00 & Ref. & \\
\hline Yes & 44 & 18.5 & 194 & 81.5 & 1.29 & $0.95-1.75$ & 0.097 \\
\hline \multicolumn{8}{|c|}{ Cataracts } \\
\hline No & 1206 & 18.1 & 5444 & 81.9 & 1.00 & Ref. & \\
\hline Yes & 56 & 16.2 & 290 & 83.8 & 1.47 & $1.11-1.95$ & 0.007 \\
\hline \multicolumn{8}{|c|}{ Others } \\
\hline No & 1209 & 18.1 & 5458 & 81.9 & 1.00 & Ref. & \\
\hline Yes & 53 & 15.9 & 280 & 84.1 & 1.01 & $0.76-1.33$ & 0.949 \\
\hline
\end{tabular}


When compared to those not engaged in physical work (retired, housework, unemployment, or physical disability), those engaged in physical work exhibited a decreased risk of cognitive impairment. In particular, participating in agricultural work exerted a significant protective effect against cognitive impairment (HR: 0.60; 95\% $\mathrm{Cl}, 0.49-0.73$ ) after adjusting for sex, ethnicity, age, BMI, education, marital status, employment type, family income, living or eating alone, participating in group activities, sleep quality, napping, smoking/passive smoking, alcohol consumption, tea consumption, water consumption, disease history, and physical exercise (Table 2). 
Table 2

HRs (95\% Cl) for associations between physical work and cognitive impairment (ZJMPHS Program, Zhejiang, China).

\begin{tabular}{|c|c|c|c|c|c|c|c|c|c|c|}
\hline & \multicolumn{2}{|c|}{$\begin{array}{l}\text { Cognitive } \\
\text { impairment }\end{array}$} & \multicolumn{2}{|c|}{$\begin{array}{l}\text { Normal } \\
\text { cognitive } \\
\text { function }\end{array}$} & \multicolumn{3}{|c|}{ Unadjusted } & \multicolumn{3}{|c|}{ Multi-adjusted a } \\
\hline & No. & $\%$ & No. & $\%$ & HR & $\begin{array}{l}95 \% \\
\mathrm{Cl}\end{array}$ & $\begin{array}{l}\mathrm{P} \\
\text { value }\end{array}$ & HR & $\begin{array}{l}95 \% \\
\mathrm{Cl}\end{array}$ & $\begin{array}{l}\mathrm{P} \\
\text { value }\end{array}$ \\
\hline \multicolumn{11}{|l|}{ Physical work } \\
\hline No & 1007 & 21.8 & 3617 & 78.2 & 1.00 & Ref. & & 1.00 & Ref. & \\
\hline Yes & 250 & 10.6 & 2099 & 89.4 & 0.44 & $\begin{array}{l}0.38- \\
0.51\end{array}$ & $<.001$ & 0.66 & $\begin{array}{l}0.55- \\
0.78\end{array}$ & $<.001$ \\
\hline \multicolumn{11}{|l|}{$\begin{array}{l}\text { Type of physical } \\
\text { work }\end{array}$} \\
\hline $\begin{array}{l}\text { Retired, } \\
\text { housework, } \\
\text { unemployment } \\
\text { or physical } \\
\text { disability }\end{array}$ & 1,007 & 21.8 & 3,617 & 78.2 & 1.00 & Ref. & & 1.00 & Ref. & \\
\hline $\begin{array}{l}\text { Mainly sedentary } \\
\text { work }\end{array}$ & 31 & 14.2 & 188 & 85.8 & 0.57 & $\begin{array}{l}0.40- \\
0.82\end{array}$ & 0.002 & 0.83 & $\begin{array}{l}0.56- \\
1.23\end{array}$ & 0.343 \\
\hline $\begin{array}{l}\text { Mainly standing } \\
\text { work }\end{array}$ & 13 & 7.3 & 164 & 92.7 & 0.37 & $\begin{array}{l}0.21- \\
0.64\end{array}$ & $<001$ & 0.64 & $\begin{array}{l}0.35^{-} \\
1.16\end{array}$ & 0.141 \\
\hline $\begin{array}{l}\text { Light physical } \\
\text { work }\end{array}$ & 33 & 12 & 242 & 88 & 0.56 & $\begin{array}{l}0.40- \\
0.80\end{array}$ & 0.001 & 0.88 & $\begin{array}{l}0.60- \\
1.30\end{array}$ & 0.531 \\
\hline $\begin{array}{l}\text { Intense physical } \\
\text { work }\end{array}$ & 4 & 6.2 & 61 & 93.8 & 0.34 & $\begin{array}{l}0.13- \\
0.90\end{array}$ & 0.030 & 0.64 & $\begin{array}{l}0.24- \\
1.75\end{array}$ & 0.389 \\
\hline Agricultural work & 169 & 10.5 & 1,444 & 89.5 & 0.41 & $\begin{array}{l}0.34- \\
0.49\end{array}$ & $<.001$ & 0.60 & $\begin{array}{l}0.49- \\
0.73\end{array}$ & $\begin{array}{l}<.001 \\
0.001\end{array}$ \\
\hline $\begin{array}{l}\text { Mode of } \\
\text { agricultural work }\end{array}$ & & & & & & - & & & & \\
\hline Manual & 165 & 10.6 & 1403 & 89.4 & 1.00 & Ref. & & & & \\
\hline $\begin{array}{l}\text { Semi-mechanized } \\
\text { or mechanized }\end{array}$ & 4 & 8.9 & 41 & 91.1 & 0.97 & $\begin{array}{l}0.34- \\
2.72\end{array}$ & 0.948 & 0.96 & $\begin{array}{l}0.32- \\
2.89\end{array}$ & 0.943 \\
\hline \multicolumn{11}{|l|}{$\begin{array}{l}\text { Type of } \\
\text { transportation to } \\
\text { work }\end{array}$} \\
\hline $\begin{array}{l}\text { Never } \\
\text { worked/work at } \\
\text { home or nearby }\end{array}$ & 1,122 & 19.8 & 4,544 & 80.2 & 1.00 & Ref. & & 1.00 & Ref. & \\
\hline
\end{tabular}

a Adjusted for sex, ethnicity, age, BMI, education, marital status, job type, family income, living or eating alone, participating in group activities, sleep quality, napping, smoking and passive smoking, alcohol consumption, tea consumption, water consumption, disease history, and physical exercise. ZJMPHS Program: Zhejiang Major Public Health Surveillance Program. 


\begin{tabular}{|c|c|c|c|c|c|c|c|c|c|c|}
\hline & \multicolumn{2}{|c|}{$\begin{array}{l}\text { Cognitive } \\
\text { impairment }\end{array}$} & \multicolumn{2}{|c|}{$\begin{array}{l}\text { Normal } \\
\text { cognitive } \\
\text { function }\end{array}$} & \multicolumn{2}{|c|}{ Unadjusted } & \multicolumn{4}{|c|}{ Multi-adjusted a } \\
\hline & No. & $\%$ & No. & $\%$ & HR & $\begin{array}{l}95 \% \\
\mathrm{Cl}\end{array}$ & $\begin{array}{l}P \\
\text { value }\end{array}$ & HR & $\begin{array}{l}95 \% \\
\mathrm{Cl}\end{array}$ & $\begin{array}{l}\mathrm{P} \\
\text { value }\end{array}$ \\
\hline Walking & 78 & 12.2 & 562 & 87.8 & 0.75 & $\begin{array}{l}0.60- \\
0.95\end{array}$ & 0.017 & 0.71 & $\begin{array}{l}0.56- \\
0.91\end{array}$ & 0.008 \\
\hline Bicycle & 25 & 8.7 & 264 & 91.3 & 0.43 & $\begin{array}{l}0.29- \\
0.64\end{array}$ & $\begin{array}{l}< \\
0.001\end{array}$ & 0.53 & $\begin{array}{l}0.35- \\
0.81\end{array}$ & 0.003 \\
\hline Motorcycle & 28 & 11.2 & 222 & 88.8 & 0.59 & $\begin{array}{l}0.40- \\
0.87\end{array}$ & 0.007 & 0.98 & $\begin{array}{l}0.64- \\
1.48\end{array}$ & 0.910 \\
\hline $\begin{array}{l}\text { Private/public } \\
\text { transportation } \\
\text { (car, subway, } \\
\text { ferry) }\end{array}$ & 8 & 5.3 & 144 & 94.7 & 0.33 & $\begin{array}{l}0.17- \\
0.67\end{array}$ & 0.002 & 0.46 & $\begin{array}{l}0.22- \\
0.98\end{array}$ & 0.045 \\
\hline $\begin{array}{l}\text { a Adjusted for } s \\
\text { participating in } \\
\text { consumption, } \\
\text { Health Surveilla }\end{array}$ & $\begin{array}{l}\text { nici } \\
\text { act } \\
\text { ons } \\
\text { ogr }\end{array}$ & $\begin{array}{l}\text { e, BN } \\
\text { sle } \\
\text { ion, }\end{array}$ & $\begin{array}{l}\text { ation } \\
\text { ity, na } \\
\text { histo }\end{array}$ & $\begin{array}{l}\text { arita } \\
\text { ng, } \\
\text { and }\end{array}$ & $\begin{array}{l}\text { job } \\
\text { anc } \\
\text { lexe }\end{array}$ & $\begin{array}{l}\text { e, fam } \\
\text { ssive } \\
\text { e. ZJı }\end{array}$ & $\begin{array}{l}\text { incom } \\
\text { loking, } \\
\text { HS Pro }\end{array}$ & $\begin{array}{l}\text { gor or } \\
\text { Zhej cor }\end{array}$ & $\begin{array}{l}\text { ing alon } \\
\text { Imption, } \\
\text { g Major }\end{array}$ & $\begin{array}{l}\text { ea } \\
\text { ublic }\end{array}$ \\
\hline
\end{tabular}

Participants who traveled to work by walking (HR: $0.71 ; 95 \% \mathrm{Cl}, 0.56-0.91)$, bicycle (HR: $0.53 ; 95 \% \mathrm{Cl}, 0.35-0.81$ ), or private/public transportation (HR: $0.46 ; 95 \% \mathrm{Cl}, 0.22-0.98$ ) had a significantly lower risk of cognitive impairment than those who had never worked, those who worked from home, and those who worked near their homes. Approximately $12 \%$ of participants with cognitive impairment and $21 \%$ of those with normal cognitive function reported engaging in physical exercise, which exerted a significant protective effect on cognitive function (HR: 0.76; 95\% $\mathrm{Cl}, 0.62-0.93$ ) after adjusting for related covariates. When compared to a lack of physical exercise, walking/tai chi ( $\mathrm{HR}: 0.75 ; 95 \% \mathrm{Cl}, 0.60-$ 0.93) exerted significant protective effects on cognitive function. In addition, exercise $1-4$ days per week (HR: $0.52 ; 95 \%$ $\mathrm{Cl}, 0.33-0.79)$ and $30-59$ minutes per session (HR: $0.61 ; 95 \% \mathrm{Cl}, 0.46-0.79)$ reduced the risk of cognitive impairment, relative to that observed for a lack of exercise (Table 3 ). 
Table 3

HRs (95\% Cl) for associations between physical exercise and cognitive impairment (ZJMPHS Program, Zhejiang, China).

\begin{tabular}{|c|c|c|c|c|c|c|c|c|c|c|}
\hline & \multicolumn{2}{|c|}{$\begin{array}{l}\text { Cognitive } \\
\text { impairment }\end{array}$} & \multicolumn{2}{|c|}{$\begin{array}{l}\text { Normal } \\
\text { cognitive } \\
\text { function }\end{array}$} & \multicolumn{2}{|c|}{ Unadjusted } & & \multicolumn{3}{|c|}{ Multi-adjusted ${ }^{\mathrm{a}}$} \\
\hline & No. & $\%$ & No. & $\%$ & HR & $\begin{array}{l}95 \% \\
\mathrm{Cl}\end{array}$ & $\begin{array}{l}P \\
\text { value }\end{array}$ & $\mathrm{HR}$ & $\begin{array}{l}95 \% \\
\mathrm{Cl}\end{array}$ & $\begin{array}{l}P \\
\text { value }\end{array}$ \\
\hline \multicolumn{11}{|l|}{ Physical exercise } \\
\hline No & 1,104 & 19.6 & 4,516 & 80.4 & 1.00 & Ref. & & 1.00 & Ref. & \\
\hline Yes & 156 & 11.4 & 1,217 & 88.6 & 0.66 & $\begin{array}{l}0.55- \\
0.79\end{array}$ & $\begin{array}{l}<.001 \\
0.00\end{array}$ & 0.76 & $\begin{array}{l}0.62- \\
0.93\end{array}$ & 0.006 \\
\hline \multicolumn{11}{|l|}{$\begin{array}{l}\text { Type of physical } \\
\text { exercise }\end{array}$} \\
\hline None & 1104 & 19.6 & 4,520 & 80.4 & 1.00 & Ref. & & 1.00 & Ref. & \\
\hline Walking/tai chi & 127 & 11.5 & 977 & 88.5 & 0.66 & $\begin{array}{l}0.54- \\
0.80\end{array}$ & $\begin{array}{l}<.001 \\
0.00\end{array}$ & 0.75 & $\begin{array}{l}0.60- \\
0.93\end{array}$ & 0.008 \\
\hline $\begin{array}{l}\text { Brisk } \\
\text { walking/yangko }\end{array}$ & 17 & 12.1 & 124 & 87.9 & 0.70 & $\begin{array}{l}0.43- \\
1.14\end{array}$ & 0.154 & 0.77 & $\begin{array}{l}0.45- \\
1.32\end{array}$ & 0.340 \\
\hline Running/aerobics & 4 & 14.3 & 24 & 85.7 & 0.67 & $\begin{array}{l}0.25- \\
1.80\end{array}$ & 0.427 & 0.72 & $\begin{array}{l}0.26- \\
1.94\end{array}$ & 0.511 \\
\hline $\begin{array}{l}\text { Public square } \\
\text { dancing }\end{array}$ & 6 & 7.4 & 75 & 92.6 & 0.69 & $\begin{array}{l}0.31- \\
1.56\end{array}$ & 0.377 & 0.92 & $\begin{array}{l}0.41- \\
2.08\end{array}$ & 0.840 \\
\hline \multicolumn{11}{|l|}{$\begin{array}{l}\text { Exercise (days } \\
\text { per week) }\end{array}$} \\
\hline None & 1104 & 19.6 & 4,521 & 80.4 & 1.00 & Ref. & & 1.00 & Ref. & \\
\hline $1-4$ days & 24 & 8 & 277 & 92 & 0.42 & $\begin{array}{l}0.28- \\
0.64\end{array}$ & $\begin{array}{l}<.001 \\
0.001\end{array}$ & 0.52 & $\begin{array}{l}0.33- \\
0.79\end{array}$ & 0.003 \\
\hline 5-7 days & 132 & 12.4 & 935 & 87.6 & 0.74 & $\begin{array}{l}0.61- \\
0.90\end{array}$ & 0.002 & 0.84 & $\begin{array}{l}0.68- \\
1.04\end{array}$ & 0.104 \\
\hline \multicolumn{11}{|l|}{$\begin{array}{l}\text { Exercise duration } \\
\text { per session (min) }\end{array}$} \\
\hline None & 1104 & 19.6 & 4,521 & 80.4 & 1.00 & Ref. & & 1.00 & Ref. & \\
\hline$<30$ & 38 & 18 & 173 & 82 & 0.93 & $\begin{array}{l}0.67- \\
1.29\end{array}$ & 0.653 & 1.14 & $\begin{array}{l}0.81- \\
1.62\end{array}$ & 0.456 \\
\hline $30-59$ & 73 & 10.4 & 628 & 89.6 & 0.56 & $\begin{array}{l}0.44- \\
0.72\end{array}$ & $\begin{array}{l}< \\
0.001\end{array}$ & 0.61 & $\begin{array}{l}0.46- \\
0.79\end{array}$ & $\begin{array}{l}< \\
0.001\end{array}$ \\
\hline
\end{tabular}

a Adjusted for sex, ethnicity, age, BMI, education, marital status, job type, family income, living or eating alone, participating in group activities, sleep quality, napping, smoking and passive smoking, alcohol consumption, tea consumption, water consumption, disease history, and agricultural work. 


\begin{tabular}{|c|c|c|c|c|c|c|c|c|c|c|}
\hline & \multicolumn{2}{|c|}{$\begin{array}{l}\text { Cognitive } \\
\text { impairment }\end{array}$} & \multicolumn{2}{|c|}{$\begin{array}{l}\text { Normal } \\
\text { cognitive } \\
\text { function }\end{array}$} & \multicolumn{3}{|c|}{ Unadjusted } & \multicolumn{3}{|c|}{ Multi-adjusted ${ }^{a}$} \\
\hline & No. & $\%$ & No. & $\%$ & HR & $\begin{array}{l}95 \% \\
\mathrm{Cl}\end{array}$ & $\begin{array}{l}P \\
\text { value }\end{array}$ & HR & $\begin{array}{l}95 \% \\
\mathrm{Cl}\end{array}$ & $\begin{array}{l}\mathrm{P} \\
\text { value }\end{array}$ \\
\hline$\geq 60$ & 43 & 9.6 & 407 & 90.4 & 0.69 & $\begin{array}{l}0.51- \\
0.95\end{array}$ & 0.021 & 0.86 & $\begin{array}{l}0.61- \\
1.20\end{array}$ & 0.367 \\
\hline
\end{tabular}

Stratified analyses revealed that physical work and exercise exerted independent effects on cognitive impairment, with HRs of $0.73(95 \% \mathrm{Cl}, 0.58-0.91)$ and $0.64(95 \% \mathrm{Cl}, 0.53-0.77)$, respectively. The estimated HR for the combination of physical work and exercise was $0.58(95 \% \mathrm{Cl}, 0.39-0.85)$, which was more than that expected from adding (0.37) and multiplying (0.47) their independent associations, suggesting an interaction between physical work and exercise under the additive and multiplicative models.

\section{Discussion}

In the present prospective study, we explored the relationship between physical activity (including physical work and exercise) and the incidence of cognitive impairment among older adults. Our results indicated that both physical work and exercise exerted significant protective effects against cognitive impairment. In particular, agricultural work and walking/tai chi were among the types of activity shown to protect against cognitive impairment.

At the end of the 2-year follow-up period, the incidence of cognitive impairment was 97.7 per 1,000 person-years, similar to that reported for the neighboring city of Shanghai (96.9/1,000 person-years) [18] yet significantly higher than the estimated incidence rates reported in other countries (from 13.2 to 76.8 per 1,000 person-years) [19-23]. This finding implies that cognitive impairment represents a much more serious problem among Chinese older adults.

In accordance with the findings of previous studies, our data provide strong evidence for the protective effect of physical activity against cognitive impairment. Physical work and exercise are the main subcategories of physical activity, and the latter is defined as "a subcategory of physical activity that is planned, structured, repetitive, and purposeful" [24]. Therefore, the effect of physical work on cognitive function was considered to differ from that of physical exercise. Notably, some studies have reported that physical work is a risk factor for cognitive impairment $[25,26]$. However, this conclusion may be inappropriate, as physical work was compared to mental labor in these previous studies. In contrast, our results verified the protective effect of physical work (especially agricultural work) against cognitive impairment. The mechanisms by which physical work reduces the risk of cognitive impairment may be analogous to those associated with physical exercise: maintenance of cerebral blood flow, improvements in aerobic capacity and cerebral nutrient supply, expansion of cognitive reserves, decreases in chronic stress, and promotion of a healthy lifestyle [27]. Moreover, our study demonstrated the additive and multiplicative effects of physical work and exercise, suggesting that those engaged in manual work should also perform regular exercise to more effectively reduce their risk of cognitive impairment and dementia.

Although the protective effect of walking on dementia remains controversial [10], several studies have noted its beneficial effects on cognitive impairment [28,29], including the present study. Although we observed protective effects 
of brisk walking, yangko, and running in the multivariable analyses, the HR suggested that they exerted no significant effects on cognitive impairment after accounting for confounding factors. As this may have been due to insufficient sample sizes in these groups, further epidemiological studies are required to determine the relationship between running/public square dancing and the risk of cognitive impairment.

Although different levels of physical exercise were shown to exert protective effects against cognitive impairment, a frequency of 1-4 days per week and a duration of 30-60 minutes were more strongly associated with a reduced risk of cognitive impairment than other levels. As reported in previous studies [30-32], we observed significant trends of increasing protection as the level of physical activity increased. Thus, based on this finding, we recommend that older adults regularly engage in moderate physical activity for $>30$ minutes $1-4$ days per week.

Our study is advantageous in that our findings are based on a rigorous prospective design, avoiding biases related to retrospective investigation of regular activities and other exposures. Of course, after onset of cognitive impairment, the normal physical work or exercise of older adults would also be restricted. In addition, physical activities were divided into physical work and exercise, helping us to quantitatively assess their independent and interactive effects on cognitive impairment. However, our study also possesses several limitations. First, some possible protective factors including several types of exercise (running and public square dancing) and types of physical work other than agricultural work exhibited no significant associations with the risk of cognitive impairment due to the limited population. Second, the study was conducted in the rural and suburban areas, and the results may not generalize to urban populations because of the different types and levels of physical work. Third, a longer follow-up period is required to avoid underestimation or overestimation of the protective effects of physical activity. Fourth, loss to follow-up and compliance are unavoidable in prospective studies, and these factors may have influenced the reliability and stability of our findings. To combat these issues, we adopted some effective measures (including mobilization, community education, etc.) to ensure that the rate of loss to follow-up remained under 10 percent. In addition, the Montreal cognitive assessment (MoCA) and other more specific scales may have been better suited for cognitive function assessment; however, scales such as MoCA are greatly influenced by the education level of participants [33]. We used the MMSE scale to measure cognitive function instead of MoCA since the education level of most participants in this study was below the primary school level.

\section{Conclusions}

Our findings demonstrated that both physical work and exercise were associated with a reduced risk of cognitive impairment among older adults. Based on our results, we recommend that older adults engage in reasonable types and appropriate intensities of physical activity [34], such as walking over 30-60 minutes per day or participating in agricultural work, in order to prevent or delay the progression of cognitive impairment. "Green" forms of transportation including walking, bicycling, or taking the bus should also be encouraged. Nonetheless, further studies are required to determine the mechanisms by which physical activity protects against cognitive impairment.

\section{List Of Abbreviations}

HR, hazard ratio

$\mathrm{Cl}$, confidence interval

$A D$, Alzheimer's disease

ZJMPHS, Zhejiang Major Public Health Surveillance

Page $14 / 17$ 
MMSE, Mini-Mental State Examination

BMI, body mass index

MoCA, Montreal cognitive assessment

$\mathrm{v>}$

\section{Declarations}

Ethics approval and consent to participate: All participants were enrolled in the Zhejiang Major Public Health Surveillance Program. Participation was voluntary, and written informed consent was obtained from each participant. The program was approved by the Ethics Committee of the Zhejiang Provincial Center for Disease Control and Prevention.

Consent for publication: Not applicable.

Availability of data and materials: All data generated or analyzed during this study are included in this published article.

Competing interests: The authors declare that they have no competing interests.

Funding: This research was supported by the National Key R \& D Program of China (2017YFC0907000), the Zhejiang Provincial Public Welfare Technology Application Research Project of China (LGF19H260003), and the Zhejiang Provincial Natural Science Foundation of China (LQ19H260001).

Authors' contributions: Conceptualization, GZ and JL; methodology, FH; software, FH; validation, FL and YZ; formal analysis, TZ; investigation, XG; resources, FH; data curation, TZ; writing-original draft preparation, FH; writing-review \& editing, GZ and JL; visualization, FL; supervision, XG; project administration, JL; funding acquisition, GZ, FH, and FL.

Acknowledgements: We would like to thank all participants in the study and the investigators from the six selected counties for their assistance with the investigation and data collection.

\section{References}

1. World Population Prospects: The 2017 Revision. https://www.un.org/development/desa/publications/worldpopulation-prospects-the-2017-revision.html. Accessed 15 June 2019.

2. Prince M, Ali GC, Guerchet M, Prina AM, Albanese E, Wu YT. Recent global trends in the prevalence and incidence of dementia, and survival with dementia. Alzheimers Res Ther. 2016; 8: 23.

3. Wu YT, Ali GC, Guerchet M, Prina AM, Chan KY, Prince M, et al. Prevalence of dementia in mainland China, Hong Kong and Taiwan: an updated systematic review and meta-analysis. Int J Epidemiol. 2018; 47: 709-19.

4. Wimo A, Guerchet M, Ali GC, Wu YT, Prina AM, Winblad B, et al. The worldwide costs of dementia 2015 and comparisons with 2010. Alzheimers Dement. 2017; 13: 1-7.

5. Parra MA, Baez S, Allegri R, Nitrini R, Lopera F, Slachevsky A, et al. Dementia in Latin America: Assessing the present and envisioning the future. Neurology. 2018; 90: 222-31.

6. Zhang Y, Gu Y, Zhang Y, Liu X, Zhang Y, Wu W, et al. Effect of sociodemographic and physical activity on cognitive function in older adults: A nationwide cross-sectional survey. Int J Geriatr Psychiatry. 2019; 34: 243-8.

7. Blondell SJ, Hammersley-Mather R, Veerman JL. Does physical activity prevent cognitive decline and dementia?: a systematic review and meta-analysis of longitudinal studies. BMC Public Health. 2014; 14: 510. 
8. Beydoun MA1, Beydoun HA, Gamaldo AA, Teel A, Zonderman AB, Wang Y. Epidemiologic studies of modifiable factors associated with cognition and dementia: systematic review and meta-analysis. BMC Public Health. 2014; 14: 643.

9. Grande G1, Vanacore N2, Maggiore L3, Cucumo V. Physical activity reduces the risk of dementia in mild cognitive impairment subjects: a cohort study. J Alzheimers Dis. 2014; 39: 833-9.

10. Lee J. The Relationship Between Physical Activity and Dementia: A Systematic Review and Meta-Analysis of Prospective Cohort Studies. J Gerontol Nurs. 2018; 44: 22-9.

11. Voss MW, Vivar C, Kramer AF, van Praag H. Bridging animal and human models of exercise-induced brain plasticity. Trends Cogn Sci. 2013; 17: 525-44.

12. Wang Z, van Praag H. Exercise and the Brain: Neurogenesis, Synaptic Plasticity, Spine Density, and Angiogenesis. In(Eds: Boecker H, Hillman C, Scheef L, Strüder H). Functional Neuroimaging in Exercise and Sport Sciences. New York: Springer; 2012.

13. World Health Organization. Noncommunicable diseases and their risk factors. Available: http://www.who.int/ncds/prevention/physical-activity/introduction/en/. Accessed 28 February 2019.

14. China's 13th five-year plan for the development of national aging undertakings and the construction of the aging care system. http://www.cncaprc.gov.cn/contents/2/179240.html. Accessed 16 March 2019.

15. Sheng Y, He F, Lin JF, Shen W, Qiu YW. Tea and Risk of Age-Related Cataracts: A Cross-Sectional Study in Zhejiang Province, China. J Epidemiol. 2016; 26: 587-92.

16. Wang Y, Eds. The rating scales for neurology. Beijing: China Friendship Publishing Company; 2005.

17. Kenneth J. Rothman, Sander Greenland, Timothy L. Lash. Modern Epidemiology. The 3rd Edition.LIPPINCOTT WILLIAMS \& WILKINS; 2008: 71-83.

18. Wang T, Xiao S, Chen K, Yang C, Dong S, Cheng Y, et al. Prevalence, Incidence, Risk and Protective Factors of Amnestic Mild Cognitive Impairment in the Elderly in Shanghai. Curr Alzheimer Res. 2017; 14: 460-6.

19. Ravaglia G, Forti P, Montesi F, Lucicesare A, Pisacane N, Rietti E, Dalmonte E, Bianchin M, Mecocci P. Mild cognitive impairment: epidemiology and dementia risk in an elderly Italian population. J Am Geriatr Soc. 2008; 56: 51-8.

20. Bae JB, Kim YJ, Han JW, Kim TH, Park JH, Lee SB, et al. Incidence of and risk factors for Alzheimer's disease and mild cognitive impairment in Korean elderly. Dement Geriatr Cogn Disord. 2015; 39: 105-15.

21. Chaves ML, Camozzato AL, Godinho C, Piazenski I, Kaye J. Incidence of mild cognitive impairment and Alzheimer disease in Southern Brazil. J Geriatr Psychiatry Neurol. 2009; 22: 181-7.

22. Caracciolo B, Palmer K, Monastero R, Winblad B, Bäckman L, Fratiglioni L. Occurrence of cognitive impairment and dementia in the community: a 9-year-long prospective study. Neurology. 2008; 70: 1778-85.

23. Manly JJ, Tang MX, Schupf N, Stern Y, Vonsattel JP, Mayeux R. Frequency and course of mild cognitive impairment in a multiethnic community. Ann Neurol. 2008; 63: 494-506.

24. World Health Organization. Global Strategy on Diet, Physical Activity and Health. https://www.who.int/dietphysicalactivity/pa/en/ . Accessed 16 March 2019.

25. Ma F, Wang T, Yin J, Bai XJ, Zhang XD, Meng J, Qu CY. A case-control study on the influencing factors to mild cognitive impairment among the community-based elderly population. Zhonghua Liu Xing Bing Xue Za Zhi. 2008: 29: 873-7.

26. Zhu YP, Chen MF, Shen BH. A prevalence study on mild cognitive impairment among elderly populations in Zhejiang province. Zhonghua Liu Xing Bing Xue Za Zhi. 2013; 34: 475-7.

27. Solfrizzi V, Capurso C, D'Introno A, Colacicco AM, Santamato A, Ranieri M, Fiore P, Capurso A, Panza F. Lifestylerelated factors in predementia and dementia syndromes. Expert Rev Neurother. 2008; 8: 133-58. 
28. Abbott RD, White LR, Ross GW, Masaki KH, Curb JD, Petrovitch H. Walking and dementia in physically capable elderly men. JAMA. 2004; 292: 1447-53.

29. Weuve J, Kang JH, Manson JE, Breteler MM, Ware JH, Grodstein F. Physical activity, including walking, and cognitive function in older women. JAMA. 2004; 292: 1454-61.

30. Laurin D, Verreault R, Lindsay J, MacPherson K, Rockwood K. Physical activity and risk of cognitive impairment and dementia in elderly persons. Arch Neurol. 2001; 58: 498-504.

31. Schlosser Covell GE, Hoffman-Snyder CR, Wellik KE, Woodruff BK, Geda YE, Caselli RJ, Demaerschalk BM, Wingerchuk DM. Physical activity level and future risk of mild cognitive impairment or dementia: a critically appraised topic. Neurologist. 2015; 19: 89-91.

32. Krell-Roesch J, Feder NT, Roberts RO, Mielke MM, Christianson TJ, Knopman DS, et al. Leisure-Time Physical Activity and the Risk of Incident Dementia: The Mayo Clinic Study of Aging. J Alzheimers Dis. 2018; 63: 149-55.

33. Cesar KG, Yassuda MS, Porto FHG, Brucki SMD, Nitrini R. MoCA Test: normative and diagnostic accuracy data for seniors with heterogeneous educational levels in Brazil. Arq Neuropsiquiatr. 2019; 77: 775-81.

34. Lam FM, Huang MZ, Liao LR, Chung RC, Kwok TC, Pang MY. Physical exercise improves strength, balance, mobility, and endurance in people with cognitive impairment and dementia: a systematic review. J Physiother. 2018; 64: 415. 